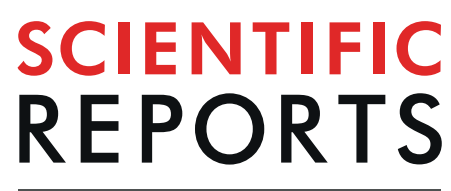

\title{
Investigation and Restoration of BEST1 Activity in Patient-derived RPEs with Dominant Mutations
}

\author{
Changyi Ji $\mathrm{i}^{1,6}$, Yao Li $\mathrm{i}^{2,6}$, Alec Kittredge ${ }^{1}$, Austin Hopiavuori ${ }^{1}$, Nancy Ward ${ }^{1}$, Peng Yao ${ }^{3}$, \\ Yohta Fukuda ${ }^{4}$, Yu Zhang ${ }^{1,2^{*}}$, Stephen H. Tsang ${ }^{5 *}$ \& Tingting Yang ${ }^{1,2^{*}}$
}

BEST1 is a $\mathrm{Ca}^{2+}$-activated $\mathrm{Cl}^{-}$channel predominantly expressed in retinal pigment epithelium (RPE), and over $\mathbf{2 5 0}$ genetic mutations in the BEST1 gene have been identified to cause retinal degenerative disorders generally known as bestrophinopathies. As most BEST1 mutations are autosomal dominant, it is of great biomedical interest to determine their disease-causing mechanisms and the therapeutic potential of gene therapy. Here, we characterized six Best vitelliform macular dystrophy (BVMD)associated BEST1 dominant mutations by documenting the patients' phenotypes, examining the subcellular localization of endogenous BEST1 and surface $\mathrm{Ca}^{2+}$-dependent $\mathrm{Cl}^{-}$currents in patientderived RPEs, and analyzing the functional influences of these mutations on BEST1 in HEK293 cells. We found that all six mutations are loss-of-function with different levels and types of deficiencies, and further demonstrated the restoration of $\mathrm{Ca}^{2+}$-dependent $\mathrm{Cl}^{-}$currents in patient-derived RPE cells by WT BEST1 gene supplementation. Importantly, BEST1 dominant and recessive mutations are both rescuable at a similar efficacy by gene augmentation via adeno-associated virus (AAV), providing a proof-of-concept for curing the vast majority of bestrophinopathies.

Genetic mutation of the human BEST1 gene causes bestrophinopathies, which consist of a spectrum of retinal degeneration disorders including Best vitelliform macular dystrophy (BVMD) $)^{1,2}$, autosomal recessive bestrophinopathy $(\mathrm{ARB})^{3}$, adult-onset vitelliform dystrophy $(\mathrm{AVMD})^{4,5}$, autosomal dominant vitreoretinochoroidopathy $(\mathrm{ADVIRC})^{6}$, and retinitis pigmentosa $(\mathrm{RP})^{7}$. BVMD, featuring an early-onset and debilitating form of central macular degeneration, is the most common bestrophinopathy. Due to abnormalities in the fluid and/or electrolyte homeostasis between the RPE and photoreceptor outer segments ${ }^{8}$, the disease leads to the formation of serous retinal detachment and lesions that resemble egg yolk, or vitelliform, while rod and cone photoreceptor function remains unaffected. All types of bestrophinopathies, except for ARB, result from autosomal dominant mutation of BEST1. Patients are susceptible to untreatable, progressive vision loss, which significantly deteriorates life quality. Therefore, understanding the mechanisms of BEST1 disease-causing mutations and designing strategies to restore the damaged cellular function are critical for developing treatments for bestrophinopathies.

The protein encoded by the BEST1 gene is a $\mathrm{Cl}^{-}$channel named BESTROPHIN1 (BEST1), which is activated in response to intracellular $\mathrm{Ca}^{2+}$ and conducts $\mathrm{Ca}^{2+}$-dependent $\mathrm{Cl}^{-}$current on the cell membrane of retinal pigment epithelium (RPE) ${ }^{1,2,9,10}$. Consistently, $\mathrm{Ca}^{2+}$-dependent $\mathrm{Cl}^{-}$current has been suggested to generate a critical visual response upon light exposure, namely light peak (LP $)^{11-13}$, which is defective in almost all BEST1-mutated patients as shown by electrooculography (EOG) ${ }^{14,15}$. This BEST1- $\mathrm{Cl}^{-}$current- LP correlation suggests gene supplementation as a promising approach for curing bestrophinopathies. Indeed, we reported that the impaired $\mathrm{Cl}^{-}$current in RPE derived from an ARB patient bearing a BEST1 recessive mutation was rescuable by baculovirus (BV) -mediated supplementation of the WT BEST1 gene ${ }^{9}$. Moreover, a recent study in canine

\footnotetext{
${ }^{1}$ Department of Pharmacology and Physiology, University of Rochester, School of Medicine and Dentistry, Rochester, NY, 14642, USA. '2 Department of Ophthalmology, Columbia University, New York, NY, 10032, USA. ${ }^{3}$ Aab Cardiovascular Research Institute, Department of Medicine, University of Rochester, School of Medicine \& Dentistry, Rochester, NY, 14586, USA. ${ }^{2}$ Division of Advance Pharmaco-Science, Graduate School of Pharmaceutical

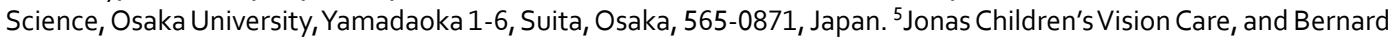
\& Shirlee Brown Glaucoma Laboratory, Departments of Ophthalmology and Pathology \& Cell Biology, Edward S. Harkness Eye Institute, Columbia Stem Cell Initiative, New York Presbyterian Hospital/Columbia University, New York, NY, 10032, USA. ${ }^{6}$ These authors contributed equally: Changyi Ji and Yao Li. *email: yz3802@cumc.columbia. edu; sht2@cumc.columbia.edu; ty2190@cumc.columbia.edu
} 
a



b

Patient 2 (R218H)

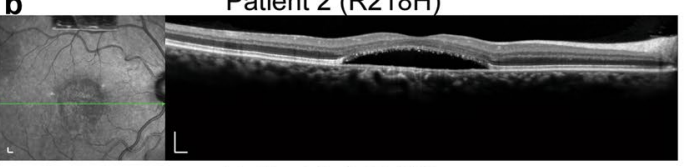

C

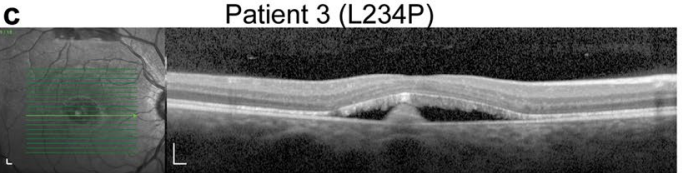

d

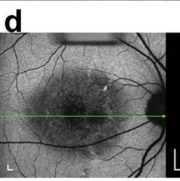

Patient 4 (A243T)

f

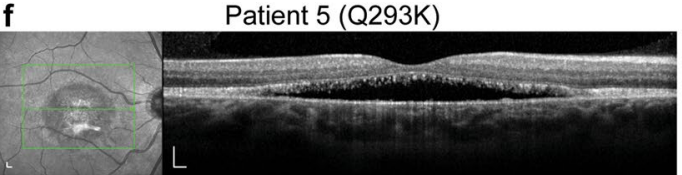

g

Patient 6 (D302A)

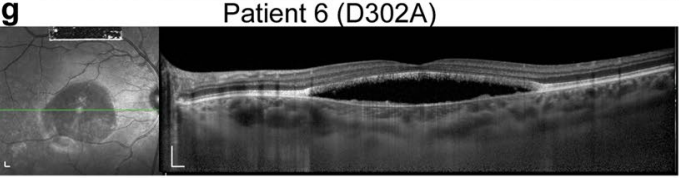

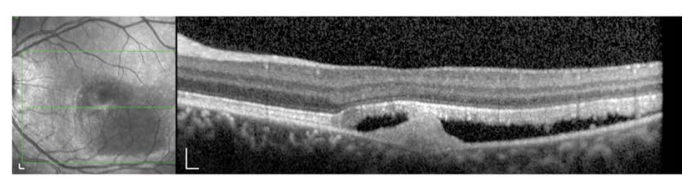
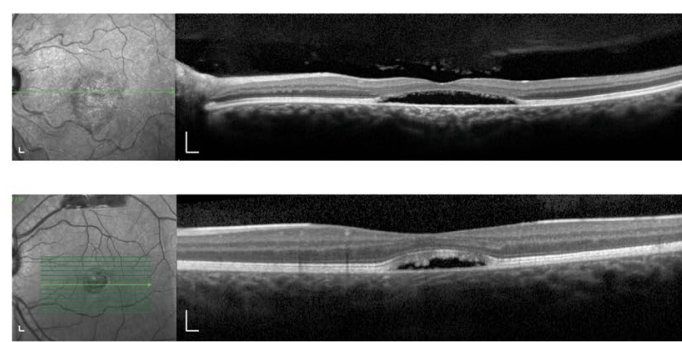

WT
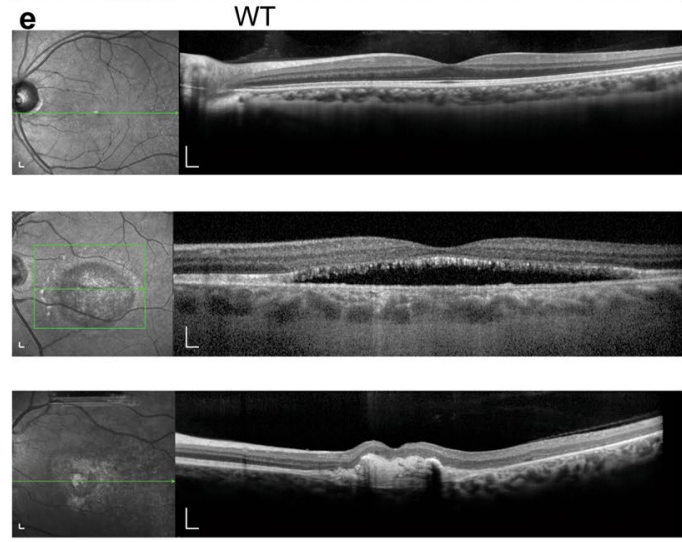

Figure 1. Clinical phenotypes of six patients with BEST1 mutations. (a-c) Fundus infrared reflectance image and Spectral Domain Optical Coherence Tomography (SDOCT) of the maculae from patient 1 (a), patient 2 (b) and patient 3 (c), right and left eyes, respectively. (d) Fundus infrared image and SDOCT of patient 4 right eye. (e) Fundus infrared reflectance image and SDOCT of a WT left eye. (f,g) Fundus infrared reflectance image and SDOCT of the maculae from patient $5(\mathbf{f})$ and patient $6(\mathbf{g})$, right and left eyes, respectively.

models demonstrated that the retinal abnormalities caused by recessive mutation of BEST1 can be corrected by adeno-associated virus (AAV) -mediated subretinal BEST1 gene augmentation ${ }^{16}$. However, the rescue efficacy of gene augmentation for BEST1 dominant mutations is still unknown. This is a very important question because firstly, most of BEST1 mutations are dominant, and secondly, it will determine whether disruption/suppression of the dominant mutant allele is necessary in therapeutic interventions. In principle, the excess of WT BEST1 could overwhelm the mutant BEST1 despite the latter being dominant over the former at a 1:1 ratio. As canines do not have BEST1 dominant mutation genotypes while Best 1 knockout mice do not show any retinal phenotype or $\mathrm{Cl}^{-}$current abnormality ${ }^{17,18}$, patient-derived RPEs offer a more relevant model for testing the rescue of BEST1 dominant mutations.

Here, we analyzed six BEST1 dominant mutations from BVMD patients, namely p.A10T, p.R218H, p.L234P, p.A243T, p.Q293K and p.D302A, using clinical examinations, patient-derived RPEs, electrophysiological recordings and structural models. Our results showed that these mutations are all loss-of-function with complete or partial deficiency of channel activity, while some of them affect the subcellular localization and/or $\mathrm{Ca}^{2+}$-sensitivity of BEST1. Remarkably, defective $\mathrm{Ca}^{2+}$-dependent $\mathrm{Cl}^{-}$currents in patient-derived RPE cells were restored by virus-mediated supplementation of the WT BEST1 gene in a time- and dose-dependent manner. Moreover, both dominant and recessive mutations of BEST1 are rescuable at a similar efficacy, and both BV and AAV can be used as the vector for gene delivery. Together, our findings underscore the great potential of gene augmentation therapy in treating bestrophinopathies, including those caused by BEST1 dominant mutations.

\section{Results}

Retinal phenotypes of six BVMD patients with different BEST1 mutations. We examined six BVMD patients from unrelated families. Generalized retinal dysfunction was found in all six patients. Fundus autofluorescence imaging and optical coherence tomography (OCT) revealed vitelliform lesions located in the subretinal space, as well as serous retinal detachments and cystic fluid in the maculae area (Fig. 1 and Supplementary Fig. S1). Unlike BEST1 recessive patients, whose electroretinography (ERG) and EOG results are significantly different from WT people ${ }^{9}$, BVMD patients display normal ERG but abnormal EOG results (Supplementary Fig. S2) ${ }^{19}$.

Patient 1, a 6-year-old otherwise healthy girl with a heterozygous c.28 G > A; p.A10T mutation, showed reduced visual acuities at 20/80 and 20/125 in the right and left eye, respectively (Table 1). Large-area, massive 


\begin{tabular}{|l|l|l|l|l|l|}
\hline Patient \# & Age & Gender & Mutation & $\begin{array}{l}\text { Vision } \\
(\text { OD })\end{array}$ & $\begin{array}{l}\text { Vision } \\
(\text { OS })\end{array}$ \\
\hline 1 & 6 & F & A10T & $20 / 80$ & $20 / 125$ \\
\hline 2 & 52 & M & R218H & $20 / 100$ & $20 / 50$ \\
\hline 3 & 7 & M & L234P & $20 / 25$ & $20 / 25$ \\
\hline 4 & 61 & F & A243T & $20 / 100$ & NA \\
\hline 5 & 44 & M & Q293K & $20 / 50$ & $20 / 50$ \\
\hline 6 & 19 & M & D302A & NA & NA \\
\hline
\end{tabular}

Table 1. Patient information. All patients have mutations in the BEST1 gene. Age: patients' age at their visit; Vision: best corrected vision; OD: right eye; OS: left eye.

vitelliform lesion was observed in the maculae of both eyes, and presented hypo-autofluorescence on fundus autofluorescence imaging. The right macula had subretinal fibrosis. OCT revealed retinal detachments in both eyes with raised fibrotic mounds in the center of the vitelliform lesion and abnormal, elongated photoreceptor outer segments. Intraretinal fluid was also observed from OCT in both eyes (Fig. 1a and Supplementary Fig. S1). Patient 2, a 52-year-old otherwise healthy man with a heterozygous c.653 G > A; p.R218H mutation, showed reduced visual acuities at 20/100 and 20/50 in the right and left eye, respectively (Table 1). OCT detected a thin photoreceptor layer in each eye, extensive subretinal serous fluid and probable vitelliform lesion (Fig. $1 \mathrm{~b}$ and Supplementary Fig. S1). Patient 3, a 7-year-old otherwise healthy boy with a heterozygous c.701 T > C; p.L234P mutation, showed reduced visual acuities at 20/25 in both eyes (Table 1). Color fundus picture and OCT showed standard boundaries-cleared yellowish vitelliform lesion in the macular area of both eyes, as well as subretinal serous fluid and retinal outer segment debris (Fig. 1c and Supplementary Fig. S1). Patient 4, a 61-year-old otherwise healthy woman with a heterozygous c.728 G > A; p.A243T mutation, showed reduced visual acuity at 20/100 in the right eye (Table 1). No data were recorded for her left eye, which has no light perception due to previous intraocular trauma with a foreign body. The vitelliform material in her right eye displayed hyper-autofluorescence in fundus autofluorescence imaging and was detected in the macular area by OCT (Fig. 1d and Supplementary Fig. S1). Her EOG testing consisted of noisy background, and there was a decrease of light rise in both eyes (Supplementary Fig. S2). Patient 5, a 44-year-old otherwise healthy man with a heterozygous c.877 C > A; p.Q293K mutation, showed reduced visual acuities at 20/50 in both eyes (Table 1). Hyper-autofluorescence of yellowish subretinal vitelliform deposits were observed in both maculae areas (Fig. If and Supplementary Fig. S1). EOG results showed loss of light rise (Supplementary Fig. S2). Patient 6 is a 19-year-old otherwise healthy man with a heterozygous c.905 A > C; p.D302A mutation, whose best corrected vision is unknown (Table 1). Vitelliform lesion with autofluoresence, serous retinal detachments and cystic fluid were found in both maculae areas (Fig. 1g and Supplementary Fig. S1).

BEST1 dominant mutations impair the channel activity of BEST1. To test the influence of the mutations on BEST1 channel activity, WT and six mutant BEST1 channels were individually introduced into HEK293 cells, which do not have any endogenous $\mathrm{Ca}^{2+}$-activated $\mathrm{Cl}^{-}$channel on the plasma membrane (Fig. 2a) ${ }^{9}$. HEK293 cells expressing BEST1 mutants displayed significantly smaller currents than WT at $1.2 \mu \mathrm{M}$ $\left[\mathrm{Ca}^{2+}\right]_{\mathrm{i}}$ (Fig. 2a and Supplementary Fig. S3), where cells expressing WT BEST1 conducted peak current amplitude $^{20}$. In particular, five mutants (A10T, R218H, L234P, Q293K and D302A) yielded tiny currents with no significant difference from untransfected cells (Fig. 2a and Supplementary Fig. S3), while the A243T mutant conducted robust currents significantly larger than those from untransfected cells but significantly smaller than those from WT BEST1 (Fig. 2a and Supplementary Fig. S3). Therefore, these six dominant mutations lead to a complete or partial loss of the BEST1 channel activity.

BEST1 dominant mutants interact with WT. The BEST1 channel is a pentamer. To test if the interaction between BEST1 monomers is affected by any of the dominant mutations, we overexpressed mutant BEST1-YFP-His and WT BEST1-CFP-Myc in HEK293 cells, followed by immunoprecipitation with an antibody against Myc and immunoblotting with antibodies against His and Myc, respectively. All six dominant mutants were expressed at similar levels to that of WT BEST1 after transient transfection, and retained the interaction with WT BEST1 (Fig. 2b).

Structural influence of BEST1 mutations. To seek the structural bases of the functional results, we analyzed a BEST1 homology model generated from the structure of chicken bestrophin1 (cBEST1) (Fig. 2c,d) ${ }^{9,21-23}$, which has $74 \%$ sequence identity with BEST1. In this model, A10, Q293 and D302 are located in the $\mathrm{Ca}^{2+}$-binding sites on the N-terminus or between S4a and S4b (Fig. 2c,d). The A10T and Q293K mutations are predicted to impair the binding of $\mathrm{Ca}^{2+}$, which is coordinated by the acidic side chains of the $\mathrm{Ca}^{2+}$-clasp and the backbone

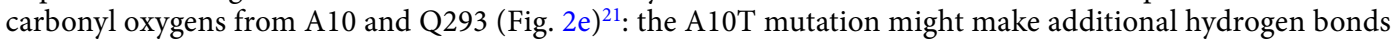
with surrounding residues including N296, one of the $\mathrm{Ca}^{2+}$ ligands; the replacement of Q293 with a lysine residue would form new interactions including a hydrogen bond with G26 and an electrostatic interaction with D303 on the $\mathrm{Ca}^{2+}$ binding loop. As D303 forms a hydrogen bond with L234 on the transmembrane helix S3b of the adjacent molecule, which contains residues controlling channel gating, the Q293K mutation also seems to have an indirect influence on channel gating. The D302A mutation changes a negative residue to a hydrophobic residue in the carboxylate loop, potentially weakening the binding of $\mathrm{Ca}^{2+}$ to the channel (Fig. 2e). Moreover, this mutation may destabilize the $\mathrm{Ca}^{2+}$ binding loop since it presumably eliminates a hydrogen bond with G26 and 


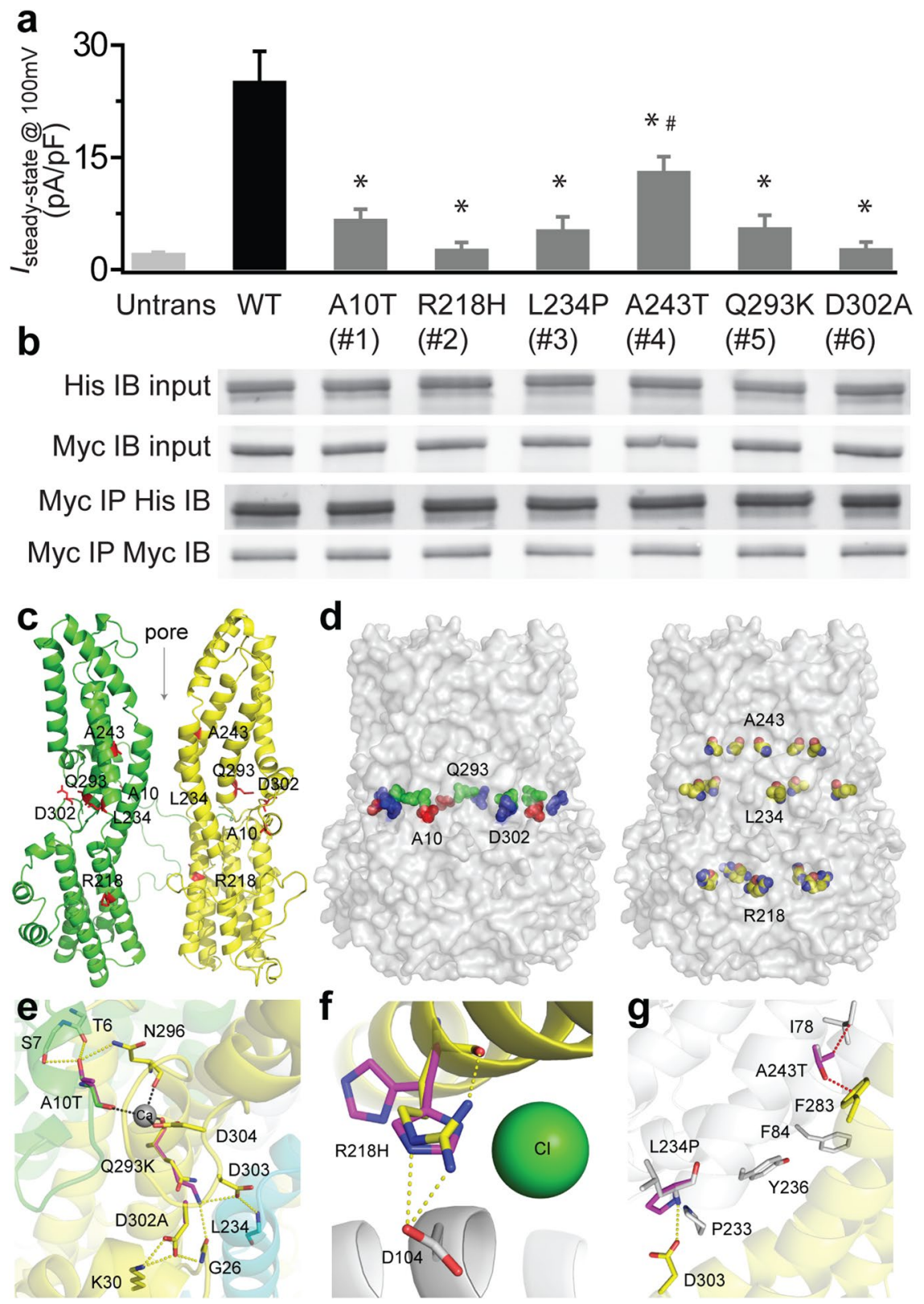

Figure 2. Disease-causing mechanisms of BEST1 mutations. (a) Bar chart showing population steady-state current densities at $100 \mathrm{mV}$ for transiently expressed BEST1 WT and mutants in HEK293 cells at $1.2 \mu \mathrm{M}$ $\left[\mathrm{Ca}^{2+}\right]_{\mathrm{i}} ; \mathrm{n}=5-6$ for each point. $* P=4 \times 10^{-5}(\mathrm{~A} 10 \mathrm{~T}), 7 \times 10^{-4}(\mathrm{R} 218 \mathrm{H}), 2 \times 10^{-3}(\mathrm{~L} 234 \mathrm{P}), 3 \times 10^{-2}(\mathrm{~A} 243 \mathrm{~T})$, $2 \times 10^{-4}(\mathrm{Q} 293 \mathrm{~K}), 2 \times 10^{-3}$ (D302A), compared to WT, respectively, and ${ }^{\#} P=6 \times 10^{-4}$ for A243T compared to untransfected cells, using two-tailed unpaired Student $t$ test. (b) WT or mutant BEST1-YFP-His was cotransfected with WT BEST1-CFP-Myc to HEK293 cells, and detected by immunoblotting directly in cell lysate (input) or after co-immunoprecipitation. The full-length blots are shown in Fig. S6. All error bars in this figure represent s.e.m. (c) Ribbon diagram of two oppositely facing $\left(144^{\circ}\right)$ protomers of a BEST1 pentamer are shown with the extracellular side on the top. The side chains of critical residues are in red. (d) Location of the patient mutations in relationship to the channel pore, as viewed from the side. A10 (red), Q293 (green) and D302 (blue) are colored differently. R218, L234 and A243 are colored by atoms. (e) Possible interactions of the mutated residues. Each monomeric unit is drawn by a different color and the mutated residues are colored in magenta. Coordination bonds and possible hydrogen bonds are illustrated by dotted black and yellow lines, respectively. (f) The effect of replacing R218 with $\mathrm{H}$. Possible conformations of $\mathrm{H} 218$ without steric hindrance are shown by magenta sticks. Each monomeric unit is drawn by a different color. Hydrogen bonds are illustrated by dotted yellow lines. (g) The effect of replacing A243 and L234. Each monomeric unit is drawn by a different color and the mutated residues are colored in magenta. Possible van der Waals contact or steric hindrance are indicated by dotted red lines. A possible hydrogen bond is illustrated by a dotted yellow line. 
an electrostatic interaction with K30. Therefore, the A10T, Q293K and D302A mutations may prohibit channel activation by diminishing $\mathrm{Ca}^{2+}$ binding, which is absolutely required for BEST1 to conduct current ${ }^{9,24}$.

R218 is localized on the alpha helix S3a (Fig. 2c,d), which falls on a putative $\mathrm{Cl}^{-}$binding site in the channel inner cavity (Fig. 2f $)^{21}$. So, the R218H mutation may decrease the local concentration of anions at the permeation pore, thereby disrupting channel activity. The model structure of the $\mathrm{R} 218 \mathrm{H}$ mutant also predicts more flexibility of H218 compared to R218 because of histidine's smaller side chain. Furthermore, H218 lacks a hydrogen bond with its own carbonyl $\mathrm{O}$ atom and is presumably located farther from D104 on the adjacent molecule compared to $\mathrm{R} 218$. Hence, the $\mathrm{R} 218 \mathrm{H}$ mutant might destabilize the local structure and weaken the interaction between monomers.

L234 and A243 are localized on the transmembrane alpha helix S3b (Fig. 2c,d), which contains multiple residues (e.g. P233 and Y236) critical for channel gating (Fig. $2 \mathrm{~g})^{25,26}$. In fact, the model structures predict that the L234P mutation cannot form a hydrogen bond with D303 from the adjacent molecule, while the A243T mutation may have steric hindrances with I78 in the same molecule and F283 from the adjacent molecule (Fig. 2g). Moreover, the L234P mutant may have a highly flexible structure around the mutation site due to consecutive proline residues.

Mutations in residues involved in $\mathrm{Ca}^{2+}$ binding disrupt membrane localization of BEST1. To directly examine the physiological impact of the six patient-specific BEST1 dominant mutations, induced pluripotent stem cells (iPSCs) were reprogrammed from the patients' skin cells and then differentiated to RPE cells (iPSC-RPEs) ${ }^{27}$. The RPE status of the cells was confirmed by morphological signatures including intracellular pigment and hexagonal shape (Fig. 3). RPE-specific marker proteins RPE65 (retinal pigment epithelium-specific $65 \mathrm{kDa}$ protein) and CRALBP (cellular retinaldehyde-binding protein) were well expressed in iPSC-RPEs derived from a BEST1 WT donor and the patients as shown by immunoblotting (Supplementary Fig. S4), confirming the mature status of all iPSC-RPEs. Moreover, all six patient-derived iPSC-RPEs showed a similar overall BEST1 expression level compared to that in iPSC-RPE derived from the BEST1 WT donor (Supplementary Fig. S4), indicating that none of the six mutations impairs the global protein expression of the channel.

We then examined the subcellular localization of BEST1 in iPSC-RPEs by immunostaining. R218H, L234P and A243T displayed normal BEST1 signals on the plasma membrane just like the WT (Fig. 3a-d). By contrast, all three mutations of residues involved in $\mathrm{Ca}^{2+}$ binding exhibited deficiency in the membrane targeting of BEST1: D302A has the strongest phenotype with a complete loss of BEST1 antibody staining signal on the plasma membrane, while A10T and Q293K both partially lost membrane localization of BEST1 (Fig. 3e-g). Consistently, immunoblotting showed decreased levels of the A10T, Q293K and D302A mutant proteins on the cell membrane (Supplementary Fig. S4).

Deficient $\mathrm{Ca}^{2+}$-dependent $\mathrm{Cl}^{-}$current in iPSC-RPEs bearing BEST1 dominant mutations. To elucidate the influences of the mutations on the physiological activity of BEST1, we measured $\mathrm{Ca}^{2+}$-dependent $\mathrm{Cl}^{-}$current in the patient-derived iPSC-RPEs by whole-cell patch clamp (Fig. 4a-f and Supplementary Fig. S5). Remarkably, tiny currents $(<6 \mathrm{pA} / \mathrm{pF})$ were detected in the A10T, R218H and D302A patient-derived iPSC-RPEs at all tested $\left[\mathrm{Ca}^{2+}\right]_{\mathrm{i}}$ (Fig. 4a,b,f and Supplementary Fig. S5), suggesting a complete loss of BEST1 channel activity in these mutants. On the other hand, robust currents were detected in the L234P, A243T and Q293K patient-derived iPSC-RPEs, but the current amplitude was significantly reduced compared to that from iPSC-RPE with WT BEST1 (Fig. 4c-e and Supplementary Fig. S5), suggesting a partial loss of function. Moreover, as currents from the A243T and Q293K iPSC-RPEs were large enough for fitting to the Hill equation, their $\mathrm{Ca}^{2+}$-sensitivity was calculated: compared to that from the WT iPSC-RPE $\left(\mathrm{K}_{\mathrm{d}}=439 \mathrm{nM}\right), \mathrm{Ca}^{2+}$-sensitivity was normal in A243T iPSC-RPE $\left(K_{d}=513 \mathrm{nM}\right)$ but significantly right shifted in Q293K iPSC-RPE ( $K_{d}=691 \mathrm{nM}$, Fig. $\left.4 \mathrm{~d}, \mathrm{e}\right)$, consistent with the structure model in which Q293 but not A243 is involved in $\mathrm{Ca}^{2+}$-binding (Fig. 2c). L234P is not expected to affect $\mathrm{Ca}^{2+}$-sensitivity, because $\mathrm{L} 234$ is localized outside of the $\mathrm{Ca}^{2+}$-clasp (Fig. 2c). For each mutation, similar electrophysiological results were obtained from two clonal iPSC-RPEs (Fig. 4g), indicating that the observed defect in $\mathrm{Ca}^{2+}$-dependent $\mathrm{Cl}^{-}$current is mutation-specific.

Taken together, our results show that the six mutations analyzed in this work can be classified into two different groups by their phenotypes: complete loss of function (A10T, R218H and D302A), and partial loss of function with normal (A243T and L234P) or decreased (Q293K) $\mathrm{Ca}^{2+}$-sensitivity.

Rescue of BEST1 dominant mutations by gene supplementation. We previously reported that the defective $\mathrm{Ca}^{2+}$-dependent $\mathrm{Cl}^{-}$current in patient-derived iPSC-RPEs carrying recessive BEST1 mutations can be rescued by baculovirus (BV)-mediated supplementation of the WT BEST1 gene ${ }^{9}$. To investigate if the $\mathrm{Ca}^{2+}$-dependent $\mathrm{Cl}^{-}$current is rescuable in iPSC-RPEs bearing BEST1 dominant mutations, WT BEST1-GFP was expressed from a BV vector in the six patient-derived iPSC-RPEs. Confocal imaging confirmed that WT BEST1-GFP is localized on the plasma membrane of all six patient-derived iPSC-RPEs (Fig. 5a), including A10T, Q293K and D302A iPSC-RPEs in which the membrane localization of endogenous BEST1 is impaired to different degrees (Fig. 3).

For electrophysiological analysis, we first utilized iPSC-RPE carrying the BEST1 R218H mutation to optimize the time course and MOI of virus infection, as $\mathrm{R} 218 \mathrm{H}$ is a null mutation with normal membrane localization of endogenous BEST1, representing a "clean" case with strong phenotypes. $\mathrm{Ca}^{2+}$-dependent $\mathrm{Cl}^{-}$current measured at $1.2 \mu \mathrm{M}\left[\mathrm{Ca}^{2+}\right]_{\mathrm{i}}$ by whole-cell patch clamp significantly increased from 24 to 48 hours, and in a dose-dependent manner at 48 hours post infection (Fig. $5 \mathrm{~b}-\mathrm{e}$ ). A complete rescue of the $\mathrm{Cl}^{-}$current at peak $\left[\mathrm{Ca}^{2+}\right]_{\mathrm{i}}$ was observed at 48 hours post infection with a minimum MOI of 100 (Fig. 5c-e, and Supplementary Fig. S5), where $\mathrm{Ca}^{2+}$-dependent $\mathrm{Cl}^{-}$currents in a full range of $\left[\mathrm{Ca}^{2+}\right]_{\mathrm{i}} \mathrm{s}$ were also fully restored (Fig. $5 \mathrm{~d}$ ). Consistently, $\mathrm{Ca}^{2+}$-dependent $\mathrm{Cl}^{-}$currents in the other five patient-derived iPSC-RPEs were all rescued to a similar level under 
BEST1
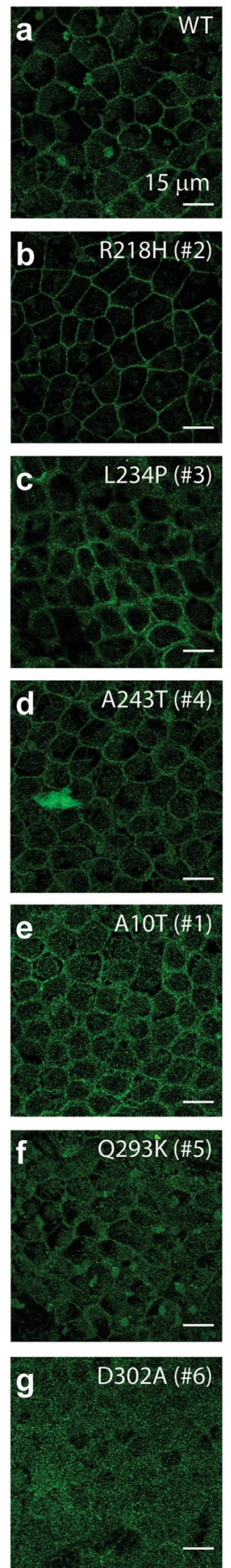

collagen IV
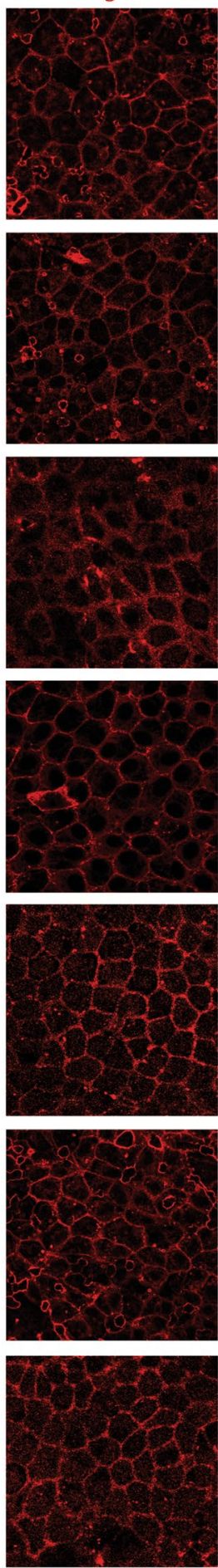

Hoechst
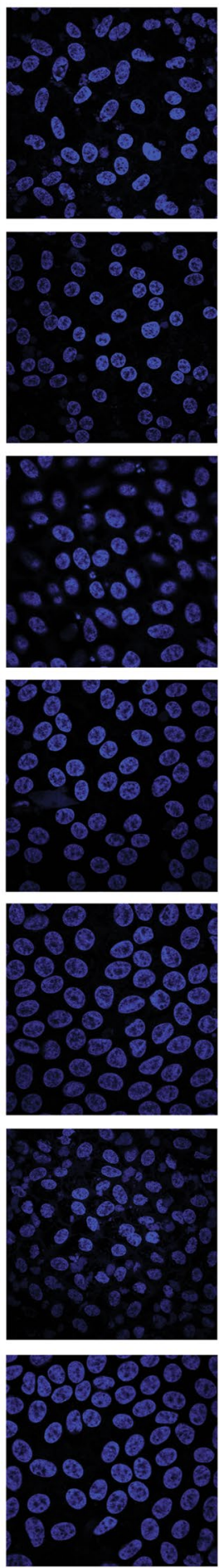

Merge
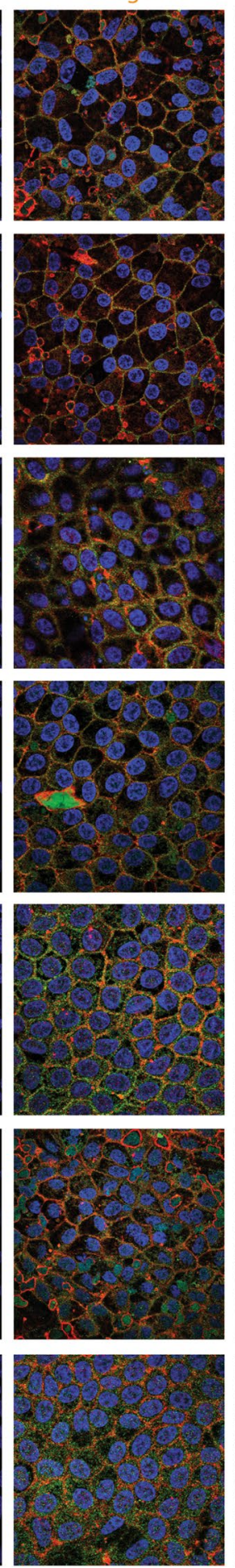

bright field
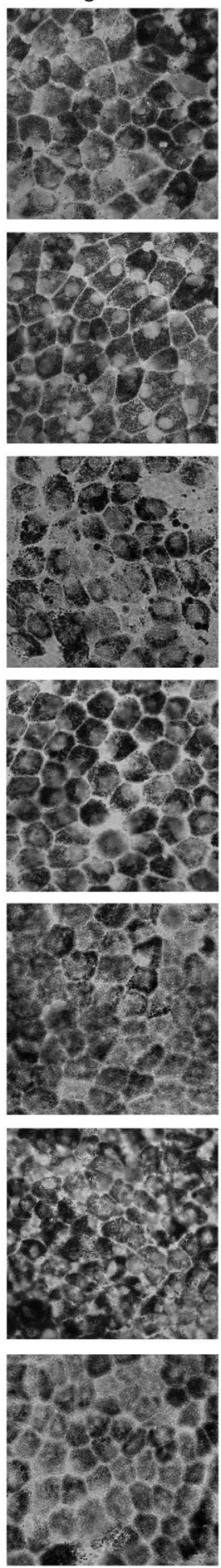

Figure 3. Subcellular localization of WT and mutant BEST1 in iPSC-RPEs. Confocal images showing the costaining of BEST1, Collagen IV and Hoechst in iPSC-RPEs derived from a WT donor or patients.

the same conditions (Fig. 5f-k), regardless of the type or level of deficiency in the endogenous BEST1 function. Immunoblotting results showed that the exogenous BEST1 expression level is comparable to that of the endogenous BEST1 (Supplementary Fig. S4).

Moreover, the rescue efficacy of $\mathrm{Ca}^{2+}$-dependent $\mathrm{Cl}^{-}$currents in iPSC-RPEs bearing BEST1 dominant mutations was comparable to that in a previously reported iPSC-RPE with a recessive P274R mutation (Fig. 5k) ${ }^{9}$. Taken together, we concluded that the defect of $\mathrm{Ca}^{2+}$-dependent $\mathrm{Cl}^{-}$conductance caused by BEST1 loss-of-function mutations, either dominant or recessive, is rescuable by BV-mediated supplementation of the WT BEST1 gene with the same dosage and time course. 


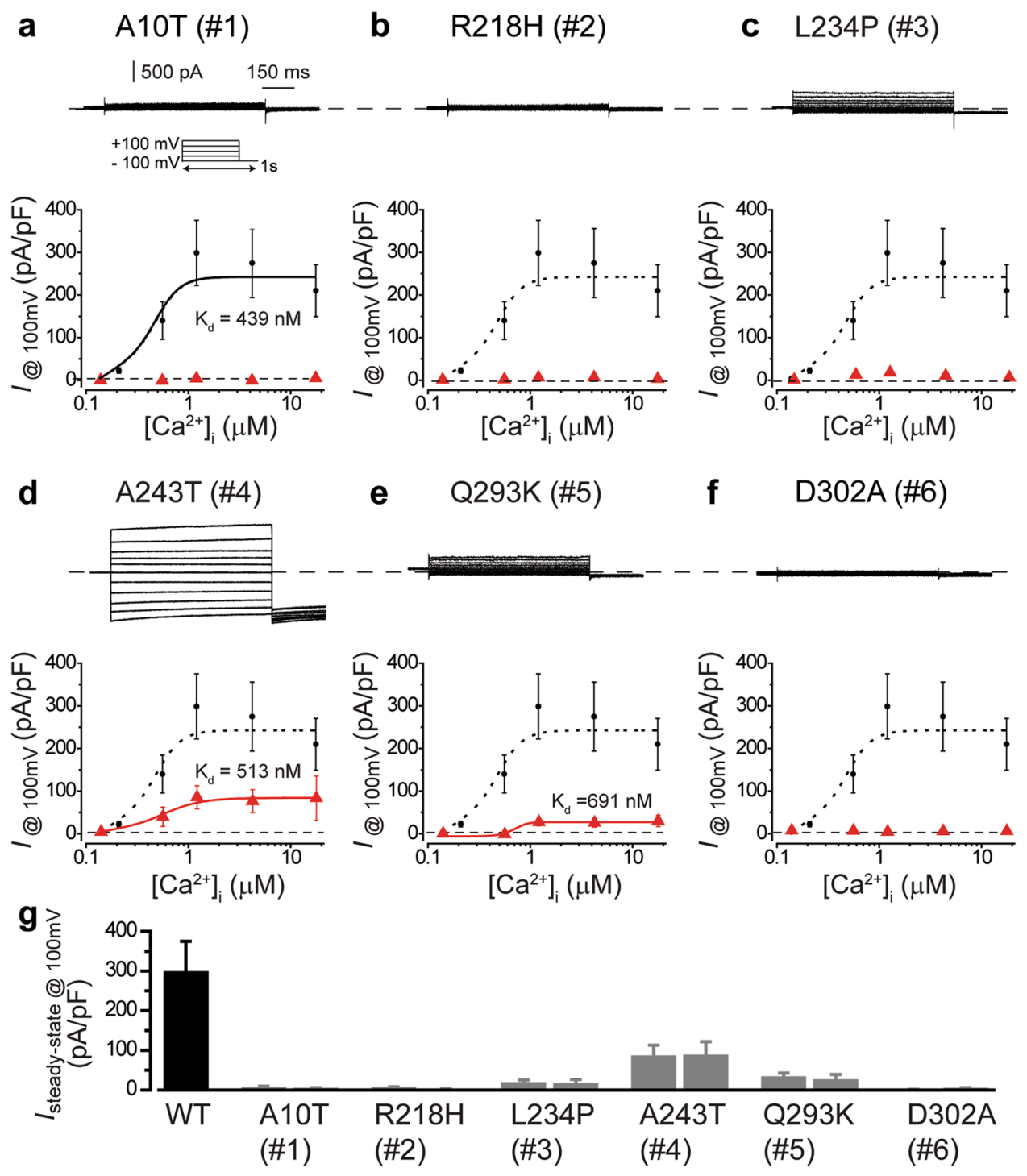

Figure 4. Surface $\mathrm{Ca}^{2+}$-dependent $\mathrm{Cl}^{-}$currents in patient-derived iPSC-RPEs. (a) $\mathrm{Ca}^{2+}$-dependent $\mathrm{Cl}^{-}$ currents measured by whole-cell patch clamp in patient-derived iPSC-RPEs bearing the mutation of A10T. Top, representative current traces recorded at $1.2 \mu \mathrm{M}\left[\mathrm{Ca}^{2+}\right]_{\mathrm{i}}$. Inset, voltage protocol used to elicit currents. Bottom, $\mathrm{Ca}^{2+}$-dependent current densities, $\mathrm{n}=5$-6 for each point, compared to WT $(\bullet)$. The WT plot was fitted to the Hill equation. (b-f) $\mathrm{Ca}^{2+}$-dependent $\mathrm{Cl}^{-}$currents measured by whole-cell patch clamp in patient-derived iPSCRPEs bearing the mutation of R218H (b), L234P (c), A243T (d), Q293K (e) and D302A (f), respectively. Top, representative current traces recorded at $1.2 \mu \mathrm{M}\left[\mathrm{Ca}^{2+}\right]_{\mathrm{i}}$. Bottom, $\mathrm{Ca}^{2+}$-dependent current densities, $\mathrm{n}=5-6$ for each point, compared to WT $(\bullet)$. The WT, A234T and Q293K plots were fitted to the Hill equation. $(\mathbf{g})$ Comparison of current densities in iPSC-RPEs with WT or mutant BEST1 at $1.2 \mu \mathrm{M}\left[\mathrm{Ca}^{2+}\right]_{\mathrm{i}}, \mathrm{n}=5-6$. Two clonal iPSC-RPEs from each patient. Black, WT. Gray, patient. All error bars in this figure represent s.e.m.

To test if BEST1 supplementation can be mediated by adeno-associated virus (AAV), which has been approved for gene therapy in the human retina ${ }^{28}$, we infected iPSC-RPEs with an AAV serotype 2 (AAV2) viral vector expressing BEST1-T2A-GFP. Consistent with the results from BV-mediated augmentation, $\mathrm{Ca}^{2+}$-dependent $\mathrm{Cl}^{-}$ currents were restored after AAV infection in iPSC-RPEs bearing either a dominant or recessive BEST1 mutation (Fig. 5i), providing a proof-of-concept for curing BEST1-associated retinal degenerative diseases in both dominant and recessive cases by AAV-mediated gene augmentation.

\section{Discussion}

Here we comprehensively examined six BEST1 dominant disease-causing mutations (A10T, R218H, L234P, A243T, Q293K and D302A) derived from BVMD patients in an interdisciplinary platform, including whole-cell patch clamp with patient-derived iPSC-RPEs and HEK293 cells expressing the mutant channels, immunodetection of endogenous BEST1 in iPSC-RPEs, structural analyses with human homology models, and virus-mediated BEST1 gene supplementation. Collectively, our results illustrate the physiological influence of these six dominant mutations on RPE surface $\mathrm{Ca}^{2+}$-dependent $\mathrm{Cl}^{-}$current and the BEST1 channel function, provide structural 


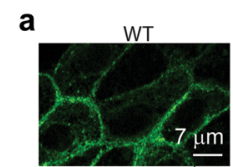

b

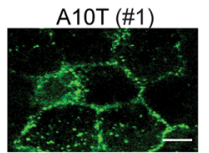$$
\text { c }
$$
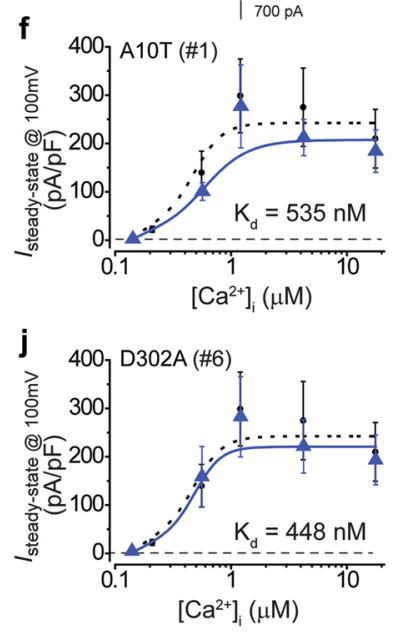

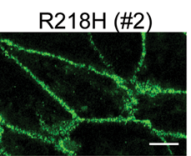

C 350
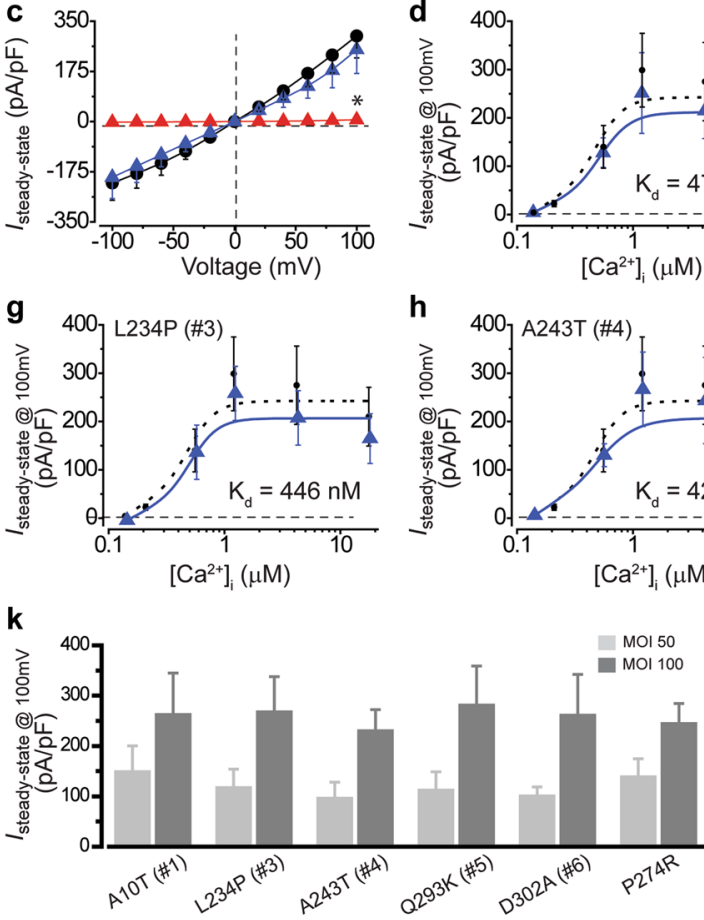

A243T (\#4)

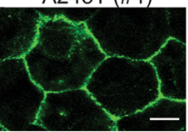

d 400

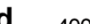

.
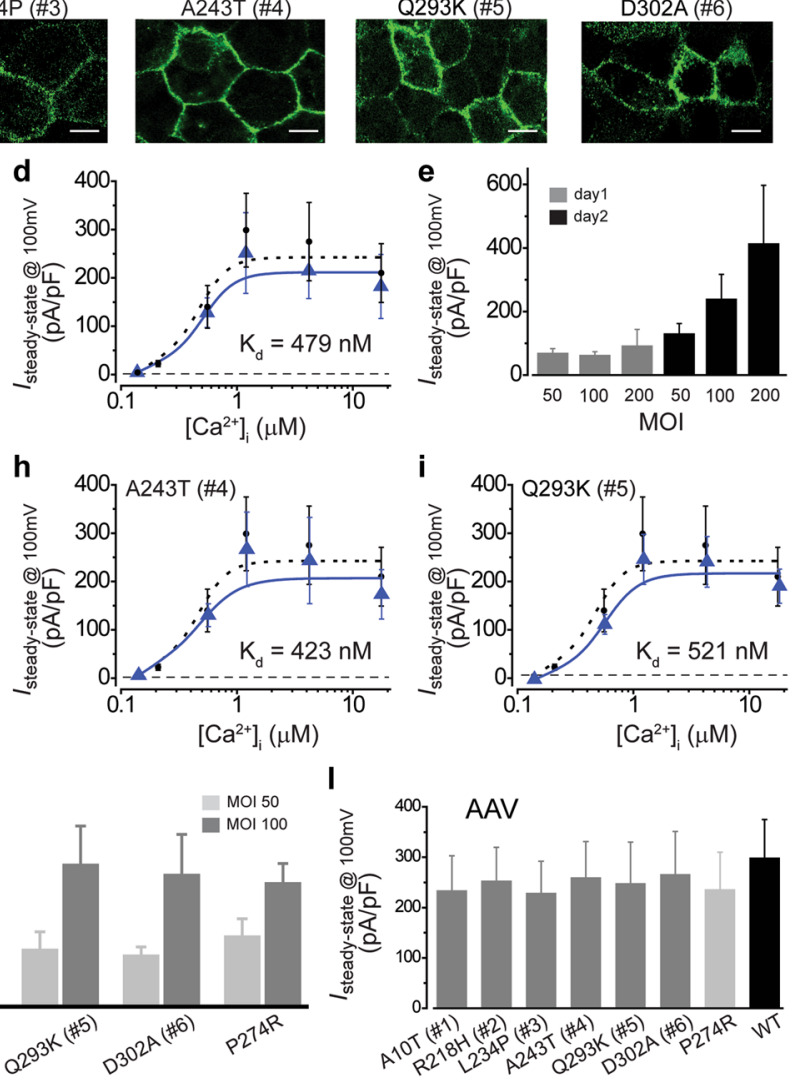

Figure 5. Rescue of patient-derived iPSC-RPEs by WT BEST1 supplementation. (a) Confocal images showing the expression of WT BEST1-GFP from BacMam virus in donor-derived iPSC-RPEs. (b) Representative current traces recorded from R218H iPSC-RPE (patient \#2) supplemented with WT BEST1-GFP at $1.2 \mu \mathrm{M}\left[\mathrm{Ca}^{2+}\right]_{\mathrm{i}}$. (c) Current densities in R218H iPSC-RPE supplemented with WT BEST1-GFP (blue triangle) at $1.2 \mu \mathrm{M}\left[\mathrm{Ca}^{2+}\right]_{\mathrm{i}}$, compared to those from un-supplemented R218H (red triangle) and WT (•) iPSC-RPEs. $\mathrm{n}=5-6$ for each point. * $P=9 \times 10^{-4}$ compared to WT, using two-tailed unpaired Student $t$ test. (d) $\mathrm{Ca}^{2+}$-dependent current densities in R218H iPSC-RPE supplemented with WT BEST1-GFP (blue triangle) compared to those from WT (•) iPSCRPE. Steady-state current density recorded at $+100 \mathrm{mV}$ plotted vs. free $\left[\mathrm{Ca}^{2+}\right]_{i} ; \mathrm{n}=5-6$ for each point. The plots were fitted to the Hill equation. (e) Current densities at $1.2 \mu \mathrm{M}\left[\mathrm{Ca}^{2+}\right]_{\mathrm{i}}$ in a second clone of R218H iPSC-RPE supplemented with different dosages of WT BEST1-GFP on BacMam viruses. $n=5-6$ for each point. $(\mathbf{f}-\mathbf{j})$ $\mathrm{Ca}^{2+}$-dependent current densities in patient-derived iPSC-RPEs bearing the mutation of A10T (f), L234P (g), A243T (h), Q293K (i) and D302A (j), supplemented with WT BEST1-GFP (blue triangle), compared to those from WT $(\bullet)$ iPSC-RPE, $n=5-6$ for each point. The plots were fitted to the Hill equation. (k) Current densities at $1.2 \mu \mathrm{M}\left[\mathrm{Ca}^{2+}\right]_{\mathrm{i}}$ in the second clones of the five BEST1 dominant iPSC-RPEs and a clone of the recessive P274R iPSC-RPE supplemented with different dosages of WT BEST1-GFP from BacMam viruses on day 2. (i) Current densities at $1.2 \mu \mathrm{M}\left[\mathrm{Ca}^{2+}\right]_{\mathrm{i}}$ in patient-derived iPSC-RPEs supplemented with WT BEST1 on AAV2 viruses. All error bars in this figure represent s.e.m.

insights into their disease-causing mechanisms, and demonstrate the rescue of BEST1 function in iPSC-RPE via gene supplementation. Notably, the diminished $\mathrm{Ca}^{2+}$-dependent $\mathrm{Cl}^{-}$currents in the R218H, L234P and A243T patient-derived iPSC-RPEs are in accord with the deficient $\mathrm{Ca}^{2+}$-stimulated $\mathrm{Cl}^{-}$secretion shown by $\mathrm{Cl}^{-}$sensitive fluorescent dyes in these cells ${ }^{29}$.

Previously, we reported that the impaired $\mathrm{Ca}^{2+}$-dependent $\mathrm{Cl}^{-}$current in iPSC-RPE derived from an ARB patient bearing a BEST1 recessive mutation (P274R) was rescuable by BV-mediated supplementation of WT BEST $1{ }^{9}$. Here, we showed that the same strategy, with both BV and AAV2, can be generally applied to restore $\mathrm{Ca}^{2+}$-dependent $\mathrm{Cl}^{-}$current impaired by BEST1 loss-of-function dominant mutations, which can be sub-classified into null (e.g. A10T, R218H and D302A), and partial deficiency with unaffected (e.g. L234P and A243T) or shifted (e.g. Q293K) $\mathrm{Ca}^{2+}$-sensitivity. Our results provide a proof-of-concept for the clinical application of BEST1 gene augmentation, but further optimization is still required: BV has not been approved for human therapy due to its strong adjuvant activity ${ }^{30}$, while the transduction efficiencies of other AAV serotypes (e.g. AAV5 and AAV8) also need to be investigated ${ }^{31}$.

The retina has been the frontier of translational gene therapy in the past 20 years. Recently, the first gene therapy drug, an AAV-based vector carrying a correct copy of the RPE65 gene, was approved by FDA for treating retinal degenerative Leber congenital amaurosis type 2 (LCA2), which is caused by recessive mutations in 
$R P E 65^{28,32-35}$. As another inherited retinal disorder clearly linked to the mutation of a single gene, bestrophinopathy represents an attractive target of gene therapy. However, since the vast majority of known BEST1 mutations are autosomal dominant, it remains a critical question whether the dominant mutant allele should be purposely suppressed during therapeutic intervention. Our results showed that virus-mediated WT BEST1 gene supplementation restores the diminished $\mathrm{Ca}^{2+}$-dependent $\mathrm{Cl}^{-}$currents in patient-derived iPSC-RPEs with the same dose- and time- dependent efficacy regardless of the mutation type (dominant vs. recessive) or deficiency level (null vs. partial), providing one of the first lines of evidence that BEST1 dominant mutations are rescuable by WT gene augmentation without the need of disrupting/suppressing the mutant allele. In agreement with our findings, a preprint by Sinha et al. showed that two more BEST1 dominant mutations, namely R218C and N296H, can be rescued by lentivirus-mediated gene augmentation in iPSC-RPE cells ${ }^{36}$. Interestingly, a third dominant mutation in that report, $\mathrm{A} 164 \mathrm{~K}$, was not responsive to gene augmentation, probably attributed to structural instability as suggested by the authors ${ }^{36}$. Nevertheless, as AAV-mediated subretinal BEST1 gene augmentation therapy has succeeded in reversing clinically detectable subretinal lesions and diffuse microdetachments in canine BEST1 recessive mutation models ${ }^{16}$, we predict that the same strategy can be applied to treat patients with either dominant or recessive BEST1 mutations as long as the mutation causes a loss-of-function.

We recently identified several gain-of-function mutations (e.g. D203A, I205T and Y236C), which significantly enhance BEST1 channel activity ${ }^{25}$. Mechanistically, these mutations dysregulate BEST1 gating at two $\mathrm{Ca}^{2+}$-dependent gates, resulting in increased channel opening ${ }^{25}$. Although the pathological basis of elevated BEST1 activity remains unclear, we speculate that knockdown or knockout of the gain-of-function mutant allele is likely necessary in addition to supplementation of the WT BEST1 gene for restoring normal BEST1 activity in these cases. However, due to the unavailability of patient-derived RPEs bearing gain-of-function mutations, whether the endogenous BEST1 protein level is negatively affected by these mutations remains unclear. Nevertheless, determining whether a patient-derived mutation causes a loss or gain of function is essential for designing the treatment strategy.

Consistent with our previous report that BEST1 is the channel responsible for $\mathrm{Ca}^{2+}$-dependent $\mathrm{Cl}^{-}$currents in $\mathrm{RPE}^{9}$, the channel activity of heterologously expressed BEST1 mutants in HEK293 cells generally reflects the integrity of $\mathrm{Ca}^{2+}$-dependent $\mathrm{Cl}^{-}$currents on the plasma membrane of the corresponding patient-derived iPSC-RPEs (Figs. 2 and 4). Although heterologous expression of WT and mutant channels in HEK293 cells is a standard and powerful approach for functional studies of BEST1, we have noticed two main limitations: firstly, the current density from HEK293 cells transiently transfected with BEST1 is significantly smaller than that from RPE cells; secondly, the $\mathrm{Ca}^{2+}$-sensitivity of BEST1-mediated currents in HEK293 cells is left-shifted compared to that in RPEs ${ }^{9,37}$. These discrepancies are likely a result of the intrinsic differences between the two cell types, rather than exogenous vs. endogenous expression of BEST1, as the $\mathrm{Ca}^{2+}$-dependent $\mathrm{Cl}^{-}$currents from supplemented WT BEST1-GFP in patient-derived iPSC-RPEs show very similar current density and $\mathrm{Ca}^{2+}$-sensitivity to those from endogenous BEST1 in WT iPSC-RPE (Figs. 4 and 5) ${ }^{9}$. We speculate that there are RPE-specific facilitating factor(s) of BEST1.

All three mutations in residues predicted to be involved in $\mathrm{Ca}^{2+}$ binding exhibited deficiency in the membrane targeting of endogenous BEST1 in iPSC-RPE, suggesting that the membrane trafficking of BEST1 may be facilitated by $\mathrm{Ca}^{2+}$ binding. However, previous studies in transiently transfected HEK293 cells showed that mutations around the $\mathrm{Ca}^{2+}$-clasp (N296L, E300Q, D301N, D302N, D303L, D304N, E306Q, and N308D) do not affect channel trafficking ${ }^{37}$. This discrepancy may be attributed to the different cell types and/or mutations in these works.

In summary, we analyzed the clinical, electrophysiological and structural impacts of six BEST1 dominant mutations, and demonstrated, among the first to our knowledge, the restoration of BEST1 function in iPSC-RPEs bearing dominant mutations by virus-mediated gene augmentation. Importantly, gene augmentation therapy also has great potential to treat other inherited disorders in the retina, such as autosomal dominant retinitis pigmentosa (adRP), which can be caused by mutations in over 25 known genes including RHO and RPE65 $5^{38}$. RHO is the most frequently mutated gene associated with adRP. AAV-mediated $R H O$ augmentation partially rescues retinal degeneration in the well-characterized $\mathrm{R} 23 \mathrm{H}$ transgenic mouse model ${ }^{39,40}$, which exhibits loss-of-function evidenced by reduced rhodopsin levels ${ }^{41-43}$. On the other hand, while RPE65 is mainly associated with LCA, a $\mathrm{D} 477 \mathrm{G}$ mutation in it has been linked to adRP ${ }^{44}$. Heterozygous RPE65 D477G knock-in mice exhibited reduced isomerase activity and delayed dark adaptation ${ }^{45}$, suggesting a loss-of-function phenotype. Therefore, our results raise the possibility of curing adRP associated with RPE65 by the FDA approved AAV-RPE65 vector without suppressing the dominant $D 477 G$ mutant allele.

\section{Methods}

Generation of human iPSC. Using the CytoTune ${ }^{\mathrm{TM}}$-iPS 2.0 Sendai Reprogramming Kit (Thermo Fisher Scientific, A16517), donor-provided skin fibroblasts were reprogrammed into pluripotent stem cells (iPSCs). Immunocytofluorescence assays were carried out following the previously published protocol to score iPSC pluripotency ${ }^{46}$. The iPSCs from all the subjects enrolled in this study were characterized by detecting four standard pluripotency markers (SSEA4, Tra-1-60, SOX2 and Nanog). Nuclei were detected by Hoechst staining. All iPSC lines were passaged every 3-6 days while maintained in mTeSR-1 medium (STEMCELL Technologies, 05850). The morphology and nuclear/cytoplasmic ratio were closely monitored to ensure the stability of the iPSC lines. All the iPSC lines were sent for karyotyping by G-banding to verify genome integrity at Cell Line Genetics (Wisconsin, USA).

Differentiation of iPSC into RPE. iPSC lines were cultured to confluence in 6-well culture dishes pretreated with 1:50 diluted matrigel (CORNING, 356230). For the first 14 days, the differentiation medium consisted of Knock-Out (KO) DMEM (Thermo Fisher Scientific, 10829018), 15\% KO serum replacement (Thermo Fisher Scientific, 10829028), 2 mM glutamine (Thermo Fisher Scientific, 35050061), 50 U/ml penicillin-streptomycin 
(Thermo Fisher Scientific, 10378016), 1\% nonessential amino acids (Thermo Fisher Scientific, 11140050), and $10 \mathrm{mM}$ nicotinamide (Sigma-Aldrich, N0636). During day 15-28 of differentiation, the differentiation medium was supplemented with $100 \mathrm{ng} / \mathrm{ml}$ human Activin-A (PeproTech, 120-14). From day 29 on, the differentiation medium without Activin-A supplementation was used again until differentiation was completed. After roughly 8-10 weeks, dispersed pigmented flat clusters were formatted and manually picked to matrigel-coated dishes. These cells were kept in RPE culture medium as previously described ${ }^{47}$. It takes another 6-8 weeks in culture for them to form a functional monolayer, which would then be ready for function assays. In addition to well-established classical mature RPE markers (Bestrophin1, CRALBP and RPE65), two more markers (PAX6 and MITF) were also used to validate the RPE fate of the cells. All iPSC-RPE cells in this study were at passage 1. DNA sequencing was used to verify genomic mutations in the mutant iPSC-RPEs.

Cell lines. Dr. David Yule at University of Rochester kindly gifted HEK293 cells. As HEK293 is included on the International Cell Line Authentication Committee's list of commonly misidentified cell lines, the cells used in this study were authenticated by short tandem repeat (STR) DNA profiling. The cells were tested negative for mycoplasma contamination, and cultured in DMEM (4.5 g/L glucose, Corning 10013CV) supplemented with 100 $\mu \mathrm{g} / \mathrm{ml}$ penicillin-streptomycin and $10 \%$ fetal bovine serum.

Electrophysiology. Using an EPC10 patch clamp amplifier (HEKA Electronics) controlled by Patchmaster (HEKA), whole-cell recordings were conducted 24-72 hours after splitting of RPE cells or transfection of HEK293 cells. $1.5 \mathrm{~mm}$ thin-walled glass with filament (WPI Instruments) were pulled and fashioned to micropipettes, and filled with internal solution containing (in $\mathrm{mM}$ ): $130 \mathrm{CsCl}, 10 \mathrm{EGTA}, 1 \mathrm{MgCl}_{2}, 2 \mathrm{MgATP}$ (added fresh), 10 HEPES ( $\mathrm{pH} 7.4$, adjusted by $\mathrm{CsOH}$ ), and $\mathrm{CaCl}_{2}$ to obtain the desired free $\mathrm{Ca}^{2+}$ concentration (maxchelator. stanford.edu/CaMgATPEGTA-TS.htm). Series resistance was usually 1.5-2.5 M $\Omega$. There was no electronic series resistance compensation. External solution contained (in $\mathrm{mM}$ ): $140 \mathrm{NaCl}, 15$ glucose, $5 \mathrm{KCl}, 2 \mathrm{CaCl}_{2}, 1 \mathrm{MgCl}_{2}$ and 10 HEPES (pH 7.4, adjusted by $\mathrm{NaOH}$ ). Solution osmolarity was between 310 and 315 . A family of step potentials $(-100$ to $+100 \mathrm{mV}$ from a holding potential of $0 \mathrm{mV})$ were used to generate $\mathrm{I}-\mathrm{V}$ curves. Currents were sampled at $25 \mathrm{kHz}$ and filtered at 5 or $10 \mathrm{kHz}$. Traces were acquired at a repetition interval of $4 \mathrm{~s}^{48}$. All experiments in this study were carried out at ambient temperature $\left(23 \pm 2^{\circ} \mathrm{C}\right)$.

Immunoblot Analysis. Cell pellets were extracted by the M-PER mammalian protein extraction reagent (Thermo Fisher Scientific, 78501) or Mem-PER Plus membrane protein extraction kit (Thermo Fisher Scientific, 89842) with proteinase inhibitors (Roche, 04693159001 ), and the protein concentration was quantified by a Bio-Rad protein reader. After denaturing at $95^{\circ} \mathrm{C}$ for $5 \mathrm{~min}$, the samples $(20 \mu \mathrm{g})$ were run on $4-15 \%$ gradient SDS-PAGE gel at room temperature, and wet transferred onto nitrocellulose membrane at $4{ }^{\circ} \mathrm{C}$. The membranes were incubated in blocking buffer containing $5 \%(\mathrm{w} / \mathrm{v})$ non-fat milk for 1 hour at room temperature, and subsequently incubated overnight at $4{ }^{\circ} \mathrm{C}$ in blocking buffer supplemented with primary antibody. Primary antibodies against the following proteins were used for immunoblotting: CRALBP (1:500 Abcam, ab15051), RPE65 (1:1,000 Novus Biologicals, NB100-355), $\beta$-actin (1:2,000 Abcam, ab8227), BESTROPHIN-1 (1:500 Novus Biologicals, NB300-164), His (1:1,000 Fisher Scientific, PA1983B) and Myc (1:1,000 Fisher Scientific, PA1981). Fluorophore-conjugated mouse and rabbit secondary antibodies (LI-COR Biosciences, 925-68070 and 92532213 , respectively) were used at a concentration of 1:10,000 and an incubation time of $1 \mathrm{~h}$ at room temperature, followed by infrared imaging.

Immunoprecipitation. HEK293 cells cultured on 6-cm dishes were co-transfected with pBacman-BEST1(WT)-CFP-myc and pBacman-BEST1(mutant or WT)-YFP-His at 1:1 ratio using PolyJet ${ }^{\mathrm{TM}}$ In Vitro DNA Transfection Reagent (SignaGen Laboratories, SL100688) following the manufacturer's standard protocol. $48 \mathrm{~h}$ later, cells were harvested by centrifugation at $1000 \mathrm{x}$ g for $5 \mathrm{~min}$ at room temperature. Cell pellets were lysed in pre-cooled lysis buffer $\left(150 \mathrm{mM} \mathrm{NaCl}, 50 \mathrm{mM}\right.$ Tris, $0.5 \%$ IGEPAL ${ }^{\circledR}$ CA-630, pH 7.4) supplemented with protease inhibitor cocktails (Roche, 04693159001 ) for $30 \mathrm{~min}$ on ice, and then centrifuged at 13,000 rpm for $12 \mathrm{~min}$ at $4^{\circ} \mathrm{C}$. The supernatants $(300 \mu \mathrm{g}$ ) was collected and mixed with $2 \mu \mathrm{g}$ Myc monoclonal antibody (Thermo Fisher Scientific, MA1-980). After rotating overnight at $4^{\circ} \mathrm{C}$, the mixture was incubated with Dynabeads $\mathrm{M}-280$ sheep anti-mouse IgG (Thermo Fisher Scientific, 11202D) for $5 \mathrm{~h}$ at $4^{\circ} \mathrm{C}$. After thorough washing of the beads, bound fractions were eluted in $1 \mathrm{x}$ SDS sample buffer (Biorad, 1610747) by heating for $10 \mathrm{~min}$ at $75^{\circ} \mathrm{C}$. Proteins were then resolved by SDS-PAGE and analyzed by immunoblotting.

Immunofluorescence. RPE cells cultured on coverslips were washed with PBS twice, and fixed in $4 \%$ sucrose and $4 \%$ paraformaldehyde at room temperature for $45 \mathrm{~min}$. The fixed cells were permeabilized in PBS containing $0.25 \%$ Triton X-100 at room temperature for $10 \mathrm{~min}$. In order to block non-specific binding sites, the samples were incubated with PBS containing 5\% BSA at room temperature for $1 \mathrm{~h}$. Primary antibodies were diluted in blocking solution as follows: mouse anti-bestrophin 1 (Novus Biologicals, NB300-164), 1:1000; rabbit anti-collagen IV (Abcam, ab6586), 1:500. The samples were incubated with primary antibody in blocking solution overnight at $4{ }^{\circ} \mathrm{C}$. The next day, the samples were washed with PBS thrice. Then, Alexa Fluor 488 conjugated donkey anti-mouse IgG (Thermo Fisher Scientific, A-21202) and Alexa Fluor 647 conjugated donkey anti-rabbit IgG (Thermo Fisher Scientific, A-31573) were diluted in blocking solution and incubated with cells at room temperature for $1 \mathrm{~h}$. Unbound secondary antibody was washed away with PBS thrice. The samples were then incubated with Hoechst 33342 diluted to $1 \mu \mathrm{g} / \mathrm{ml}$ in PBS at room temperature for $10 \mathrm{~min}$. After thorough washing, coverslips were mounted onto ProLong Diamond Antifade Mountant (Thermo Fisher Scientific, P36966). An Olympus laser scanning confocal microscope was used to acquire images, which were then processed in Fiji (https://fiji.sc/). Background was subtracted by the rolling ball method in Fiji with a radius of 50 pixels. 
Virus. BacMam baculovirus bearing BEST1-GFP was made in-house as previously described ${ }^{49}$, and added to RPE culture medium at desired MOI (50-200). High titer AAV2 virus $\left(1 \times 10^{12} \mathrm{GC} / \mathrm{ml}\right)$ bearing a CMV promoter driven BEST1-T2A-GFP expression cassette was purchased from Applied Biological Materials.

Molecular cloning. Point mutations in BEST1 were made by site-directed mutagenesis PCR with the In-fusion Cloning Kit (Clontech). All constructs were fully sequenced.

Transfection. $\quad 20-24 \mathrm{~h}$ before transfection, HEK293 cells were lifted by incubation with $0.25 \%$ trypsin at room temperature for $5 \mathrm{~min}$, and split into fresh $3.5-\mathrm{cm}$ culture dishes at proximately $50 \%$ confluency. PolyJet transfection reagent (SignaGen SL100688) was utilized to transfect HEK293 cells with plasmids bearing the WT BEST1 or desired mutant $(1 \mu \mathrm{g}) .6-8 \mathrm{~h}$ later, the transfection mix was removed, and cells were rinsed with PBS once and cultured in supplemented DMEM. $24 \mathrm{~h}$ post transfection, cells were lifted again by trypsin treatment and split onto fibronectin-coated glass coverslips for patch clamp ${ }^{50}$.

Electrophysiological data and statistical analyses. With Patchmaster (HEKA), Microsoft Excel and Origin, patch clamp data were analyzed off-line. Statistical analyses were conducted using built-in functions in Origin. For comparisons between two groups, statistically significant differences between means $(P<0.05)$ were determined using Student's $t$ test. Data are presented as means \pm s.e.m $\mathrm{m}^{51}$.

Homology modeling of human BEST1. A homology model for BEST1 was generated using the Swiss-Model server from the chicken Best 1 crustal structure ${ }^{21}$. All figures were made in PyMOL.

Patients and clinical analysis. The healthy control donor (WT BEST1) and patients (mutant BEST1) all underwent a complete ophthalmic examination by a retinal physician in the Department of Ophthalmology, Columbia University Medical Center/New York Presbyterian Hospital. This included funduscopy, best-corrected visual acuity, and slit-lamp biomicroscopy. Patients underwent OCT and color fundus photography ${ }^{52,53}$. Skin biopsy samples were obtained from the healthy control donor and patients, and processed and cultured as previously described $^{46}$. For these procedures, all of which were approved by Columbia University Institutional Review Board (IRB) protocol AAAF1849, patients 2, 4-6 and the parent(s)/legal guardian(s) of patients 1 and 3 provided written informed consent. All methods were performed in accordance with the relevant regulations and guidelines.

\section{Data availability}

Data supporting the findings of this manuscript are available from the corresponding author upon reasonable request.

Received: 24 June 2019; Accepted: 19 November 2019;

Published online: 13 December 2019

\section{References}

1. Marquardt, A. et al. Mutations in a novel gene, VMD2, encoding a protein of unknown properties cause juvenile-onset vitelliform macular dystrophy (Best's disease). Human molecular genetics 7, 1517-1525 (1998).

2. Petrukhin, K. et al. Identification of the gene responsible for Best macular dystrophy. Nature genetics 19, 241-247 (1998).

3. Burgess, R. et al. Biallelic mutation of BEST1 causes a distinct retinopathy in humans. American journal of human genetics 82, 19-31 (2008).

4. Allikmets, R. et al. Evaluation of the Best disease gene in patients with age-related macular degeneration and other maculopathies. Hum Genet 104, 449-453 (1999).

5. Kramer, F. et al. Mutations in the VMD2 gene are associated with juvenile-onset vitelliform macular dystrophy (Best disease) and adult vitelliform macular dystrophy but not age-related macular degeneration. Eur J Hum Genet 8, 286-292 (2000).

6. Yardley, J. et al. Mutations of VMD2 splicing regulators cause nanophthalmos and autosomal dominant vitreoretinochoroidopathy (ADVIRC). Investigative ophthalmology \& visual science 45, 3683-3689 (2004).

7. Davidson, A. E. et al. Missense mutations in a retinal pigment epithelium protein, bestrophin-1, cause retinitis pigmentosa. American journal of human genetics 85, 581-592 (2009).

8. Yang, T., Justus, S., Li, Y. \& Tsang, S. H. BEST1: the Best Target for Gene and Cell Therapies. Molecular therapy: the journal of the American Society of Gene Therapy 23, 1805-1809 (2015).

9. Li, Y. et al. Patient-specific mutations impair BESTROPHIN1's essential role in mediating Ca2+-dependent Cl-currents in human RPE. Elife, https://doi.org/10.7554/eLife.29914 (2017).

10. Marmorstein, A. D. et al. Bestrophin, the product of the Best vitelliform macular dystrophy gene (VMD2), localizes to the basolateral plasma membrane of the retinal pigment epithelium. Proc Natl Acad Sci USA 97, 12758-12763 (2000).

11. Fujii, S., Gallemore, R. P., Hughes, B. A. \& Steinberg, R. H. Direct evidence for a basolateral membrane Cl- conductance in toad retinal pigment epithelium. The American journal of physiology 262, C374-383 (1992).

12. Gallemore, R. P. \& Steinberg, R. H. Effects of DIDS on the chick retinal pigment epithelium. II. Mechanism of the light peak and other responses originating at the basal membrane. J Neurosci 9, 1977-1984 (1989).

13. Gallemore, R. P. \& Steinberg, R. H. Light-evoked modulation of basolateral membrane Cl- conductance in chick retinal pigment epithelium: the light peak and fast oscillation. Journal of neurophysiology 70, 1669-1680 (1993).

14. Boon, C. J. et al. The spectrum of ocular phenotypes caused by mutations in the BEST1 gene. Progress in retinal and eye research $\mathbf{2 8}$, 187-205 (2009).

15. Marmorstein, A. D., Cross, H. E. \& Peachey, N. S. Functional roles of bestrophins in ocular epithelia. Progress in retinal and eye research 28, 206-226 (2009).

16. Guziewicz, K. E. et al. BEST1 gene therapy corrects a diffuse retina-wide microdetachment modulated by light exposure. Proc Natl Acad Sci USA 115, E2839-E2848 (2018).

17. Marmorstein, L. Y. et al. The light peak of the electroretinogram is dependent on voltage-gated calcium channels and antagonized by bestrophin (best-1). J Gen Physiol 127, 577-589 (2006).

18. Milenkovic, A. et al. Bestrophin 1 is indispensable for volume regulation in human retinal pigment epithelium cells. Proc Natl Acad Sci USA 112, E2630-2639 (2015).

19. Johnson, A. A. et al. Bestrophin 1 and retinal disease. Progress in retinal and eye research. https://doi.org/10.1016/j. preteyeres.2017.01.006 (2017). 
20. Hartzell, H. C., Qu, Z., Yu, K., Xiao, Q. \& Chien, L. T. Molecular physiology of bestrophins: multifunctional membrane proteins linked to best disease and other retinopathies. Physiol Rev 88, 639-672 (2008).

21. Kane Dickson, V., Pedi, L. \& Long, S. B. Structure and insights into the function of a $\mathrm{Ca}(2+)$-activated $\mathrm{Cl}(-)$ channel. Nature 516, 213-218 (2014).

22. Yang, T. et al. Structure and selectivity in bestrophin ion channels. Science 346, 355-359 (2014).

23. Zhang, Y. et al. ATP activates bestrophin ion channels through direct interaction. Nature communications 9, 3126 (2018).

24. Sun, H., Tsunenari, T., Yau, K. W. \& Nathans, J. The vitelliform macular dystrophy protein defines a new family of chloride channels. Proc Natl Acad Sci USA 99, 4008-4013 (2002).

25. Ji, C. et al. Dual Ca(2+)-dependent gates in human Bestrophin1 underlie disease-causing mechanisms of gain-of-function mutations. Commun Biol 2, 240 (2019).

26. Miller, A. N., Vaisey, G. \& Long, S. B. Molecular mechanisms of gating in the calcium-activated chloride channel bestrophin. Elife, 8, https://doi.org/10.7554/eLife.43231 (2019).

27. Kittredge, A., Ji, C., Zhang, Y. \& Yang, T. Differentiation, Maintenance, and Analysis of Human Retinal Pigment Epithelium Cells: A Disease-in-a-dish Model for BEST1 Mutations. Journal of visualized experiments: JoVE, https://doi.org/10.3791/57791 (2018).

28. Russell, S. et al. Efficacy and safety of voretigene neparvovec (AAV2-hRPE65v2) in patients with RPE65-mediated inherited retinal dystrophy: a randomised, controlled, open-label, phase 3 trial. Lancet 390, 849-860 (2017).

29. Moshfegh, Y. et al. BESTROPHIN1 mutations cause defective chloride conductance in patient stem cell-derived RPE. Human molecular genetics 25, 2672-2680 (2016).

30. Ono, C., Okamoto, T., Abe, T. \& Matsuura, Y. Baculovirus as a Tool for Gene Delivery and Gene Therapy. Viruses, https://doi. org/10.3390/v10090510 (2018).

31. Trapani, I. \& Auricchio, A. Seeing the Light after 25 Years of Retinal Gene Therapy. Trends Mol Med 24, 669-681 (2018).

32. Jacobson, S. G. et al. Gene therapy for leber congenital amaurosis caused by RPE65 mutations: safety and efficacy in 15 children and adults followed up to 3 years. Arch Ophthalmol 130, 9-24 (2012).

33. Testa, F. et al. Three-year follow-up after unilateral subretinal delivery of adeno-associated virus in patients with Leber congenital Amaurosis type 2. Ophthalmology 120, 1283-1291 (2013).

34. Bainbridge, J. W. et al. Effect of gene therapy on visual function in Leber's congenital amaurosis. The New England journal of medicine 358, 2231-2239 (2008).

35. Bennett, J. et al. Safety and durability of effect of contralateral-eye administration of AAV2 gene therapy in patients with childhoodonset blindness caused by RPE65 mutations: a follow-on phase 1 trial. Lancet 388, 661-672 (2016).

36. Sinha, D. et al. Human iPSC modeling elucidates mutation-specific responses to gene therapy in a genotypically diverse dominant maculopathy. bioRxiv, https://doi.org/10.1101/796581

37. Xiao, Q., Prussia, A., Yu, K., Cui, Y. Y. \& Hartzell, H. C. Regulation of bestrophin Cl channels by calcium: role of the C terminus. J Gen Physiol 132, 681-692 (2008).

38. Daiger, S. P., Bowne, S. J. \& Sullivan, L. S. Genes and Mutations Causing Autosomal Dominant Retinitis Pigmentosa. Cold Spring Harbor perspectives in medicine. https://doi.org/10.1101/cshperspect.a017129 (2014).

39. Lewin, A. S., Rossmiller, B. \& Mao, H. Gene augmentation for adRP mutations in RHO. Cold Spring Harbor perspectives in medicine. https://doi.org/10.1101/cshperspect.a017400 (2014)

40. Mao, H. et al. AAV delivery of wild-type rhodopsin preserves retinal function in a mouse model of autosomal dominant retinitis pigmentosa. Human gene therapy 22, 567-575 (2011).

41. Wu, T. H. et al. Opsin localization and rhodopsin photochemistry in a transgenic mouse model of retinitis pigmentosa. Neuroscience 87, 709-717 (1998).

42. Noorwez, S. M., Sama, R. R. \& Kaushal, S. Calnexin improves the folding efficiency of mutant rhodopsin in the presence of pharmacological chaperone 11-cis-retinal. The Journal of biological chemistry 284, 33333-33342 (2009).

43. Kemp, C. M., Jacobson, S. G., Roman, A. J., Sung, C. H. \& Nathans, J. Abnormal rod dark adaptation in autosomal dominant retinitis pigmentosa with proline-23-histidine rhodopsin mutation. Am J Ophthalmol 113, 165-174 (1992).

44. Bowne, S. J. et al. A dominant mutation in RPE65 identified by whole-exome sequencing causes retinitis pigmentosa with choroidal involvement. Eur J Hum Genet 19, 1074-1081 (2011).

45. Shin, Y., Moiseyev, G., Chakraborty, D. \& Ma, J. X. A Dominant Mutation in Rpe65, D477G, Delays Dark Adaptation and Disturbs the Visual Cycle in the Mutant Knock-In Mice. The American journal of pathology 187, 517-527 (2017).

46. Li, Y., Nguyen, H. V. \& Tsang, S. H. Skin Biopsy and Patient-Specific Stem Cell Lines. Methods Mol Biol 1353, 77-88 (2016).

47. Maminishkis, A. et al. Confluent monolayers of cultured human fetal retinal pigment epithelium exhibit morphology and physiology of native tissue. Investigative ophthalmology \& visual science 47, 3612-3624 (2006).

48. Yang, T., Hendrickson, W. A. \& Colecraft, H. M. Preassociated apocalmodulin mediates Ca2+-dependent sensitization of activation and inactivation of TMEM16A/16B Ca2+-gated Cl- channels. Proc Natl Acad Sci USA 111, 18213-18218 (2014)

49. Goehring, A. et al. Screening and large-scale expression of membrane proteins in mammalian cells for structural studies. Nature protocols $9,2574-2585(2014)$

50. Yang, T., He, L. L., Chen, M., Fang, K. \& Colecraft, H. M. Bio-inspired voltage-dependent calcium channel blockers. Nature communications 4, 2540 (2013).

51. Yang, T., Suhail, Y., Dalton, S., Kernan, T. \& Colecraft, H. M. Genetically encoded molecules for inducibly inactivating CaV channels. Nat Chem Biol 3, 795-804 (2007).

52. Kohl, S. et al. Mutations in the unfolded protein response regulator ATF6 cause the cone dysfunction disorder achromatopsia. Nature genetics 47, 757-765 (2015).

53. McCulloch, D. L. et al. ISCEV Standard for full-field clinical electroretinography (2015 update). Documenta ophthalmologica. Advances in ophthalmology 130, 1-12 (2015).

\section{Acknowledgements}

The Jonas Children's Vision Care is supported by NYSTEM (C32590GG), NIH (EY019007, EY018213, EY026682), National Cancer Institute Core (5P30CA013696), Foundation Fighting Blindness (TA-NMT-0116-0692-COLU), the Research to Prevent Blindness (RPB) Physician-Scientist Award, and unrestricted funds from RPB. Y. F. was funded by Grant-in-Aid for Young Scientists (B) from the Japan Society for the Promotion of Science (17K17862). This work was supported by NIH grant EY025290 and GM127652 to T.Y.

\section{Author contributions}

C.J. performed patch clamp, confocal microscopy, immunoblotting, co-IP and analyzed data, Y.L. generated iPSC-RPE cells and helped write the paper; A.K. generated constructs; A.H. maintained RPE culture; N.W. made baculoviruses; P.Y. edited the manuscript; Y.F. analyzed structural data, made figures and helped write the paper; Y.Z. performed immunoblotting and co-IP, designed experiments and wrote the paper; S.H.T. cared for BEST1 patients and performed skin biopsies; T.Y. designed experiments, analyzed data, made figures and wrote the paper. 


\section{Competing interests}

The authors declare competing interests in relation to the work described: Provisional patent application. Patent applicant (whether author or institution): University of Rochester and Columbia University. Name of inventor(s): Tingting Yang, Stephen H. Tsang and Yu Zhang. Application number: Provisional Patent Application No. 62/785,739 and No. 62/833,069. Status of application: Provisional patent application. Specific aspect of manuscript covered in patent application: Gene therapy for BEST1 dominant mutations, including all the six mutations in the manuscript.

\section{Additional information}

Supplementary information is available for this paper at https://doi.org/10.1038/s41598-019-54892-7.

Correspondence and requests for materials should be addressed to Y.Z., S.H.T. or T.Y.

Reprints and permissions information is available at www.nature.com/reprints.

Publisher's note Springer Nature remains neutral with regard to jurisdictional claims in published maps and institutional affiliations.

(c) (i) Open Access This article is licensed under a Creative Commons Attribution 4.0 International License, which permits use, sharing, adaptation, distribution and reproduction in any medium or format, as long as you give appropriate credit to the original author(s) and the source, provide a link to the Creative Commons license, and indicate if changes were made. The images or other third party material in this article are included in the article's Creative Commons license, unless indicated otherwise in a credit line to the material. If material is not included in the article's Creative Commons license and your intended use is not permitted by statutory regulation or exceeds the permitted use, you will need to obtain permission directly from the copyright holder. To view a copy of this license, visit http://creativecommons.org/licenses/by/4.0/.

(C) The Author(s) 2019 